\title{
Femoral Neuropathy and Meralgia Paresthetica Secondary to an Iliacus Hematoma
}

\author{
Tae Im Yi, M.D., Tae Hee Yoon, M.D., Joo Sup Kim, M.D., Ga Eun Lee, M.D., Bo Ra Kim, M.D.
}

Department of Rehabilitation Medicine, Daejin Medical Center Bundang Jesaeng General Hospital, Seongnam 463-774, Korea

Compressive femoral and lateral femoral cutaneous neuropathies from an iliacus hematoma are unusual presentation. We report a case of a 16-year-old boy who developed right femoral and lateral femoral cutaneous neuropathies as a complication of traumatic ipsilateral iliacus hematoma formation. The patient complained of numbness in the right thigh and calf as well as right leg weakness, and pain in the right inguinal area. Nerve conduction study and needle electromyography identified the neuropathies. After the electrodiagnostic studies, the pelvic bone MRI revealed a large, $9 \times 5 \times 4.5 \mathrm{~cm}$ right iliacus hematoma. As a result, diagnosis of a right iliacus hematoma compressing the femoral and lateral femoral cutaneous nerves was made, and the patient underwent an operation to remove the hematoma. Symptoms and neurological signs showed notable improvement after surgical decompression. Subsequent follow-up electrodiagnostic studies after 11 weeks demonstrated regeneration evidence.

Key Words Femoral neuropathy, Meralgia paresthetica, Iliacus hematoma

\section{INTRODUCTION}

Femoral neuropathy, an uncommon peripheral neuropathy, may show symptoms such as atrophy of quadriceps muscle, weakness of hip flexion and knee extension, decreased or absent knee-jerk, hypesthesia in the anteromedial thigh and the medial side of lower leg, and pain in the inguinal area. It can be caused by pelvic mass, retroperitoneal bleeding induced by anticoagulants, lithotomy

Received November 18, 2010; Accepted April 19, 2011

Corresponding author: Tae Hee Yoon

Department of Rehabilitation Medicine, Daejin Medical Center Bundang Jesaeng General Hospital, 255-2, Seohyeon-dong, Bundang-gu, Seongnam 463-774, Korea

Tel: +82-31-779-0395, Fax: +82-31-779-0635, E-mail: thyoon79@gmail. com

(c) This is an open-access article distributed under the terms of the Creative Commons Attribution Non-Commercial License (http:// creativecommons.org/licenses/by-nc/3.0) which permits unrestricted noncommercial use, distribution, and reproduction in any medium, provided the original work is properly cited.

Copyright $\odot 2012$ by Korean Academy of Rehabilitation Medicine position for a long time, positions causing overstretching of the femoral nerve, trauma, infection, systemic diseases such as diabetes or alcoholism, and radiation injury. The most common cause is the compression of nerves around the inguinal area. ${ }^{1,2}$

Meralgia paresthetica occurs when lateral femoral cutaneous nerves are compressed by mechanical causes such as tight clothes, obesity, pregnancy and abdominal mass. Other possible causes include artificial factors such as bone graft using iliac bone and inguinal hernia repair, trauma and metabolic or toxic diseases. It shows characteristic clinical patterns such as hypesthesia, numbness, paresthesia and pain in the anterolateral thigh where nerves distribute. ${ }^{3,4}$

Our case is a peculiar one of neuropathy that occurred as a right iliacus hematoma caused by a trauma simultaneously compressing both femoral and lateral femoral cutaneous nerve. Some cases of single femoral neuropa- 

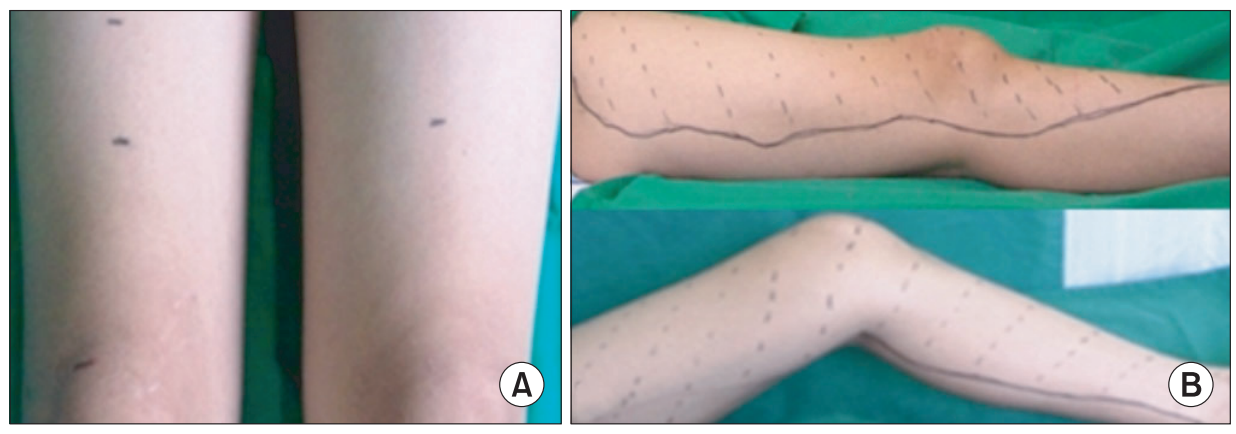

Fig. 1. Circumferences measured $10 \mathrm{~cm}$ above the superior patellar border show notable difference. The right side is $40 \mathrm{~cm}$, while the left is $46 \mathrm{~cm}$ (frontal view) (A). The dotted area is the area of hypesthesia (lateral and medial view) (B).

thy have been reported, ${ }^{5}$ but a case like ours with combined damages is rare.

Thus, we identified the atypical case accompanied simultaneously with both femoral neuropathy and meralgia paresthetica caused by iliacus hematoma through related neurologic examinations, magnetic resonance imaging (MRI) and electrodiagnostic studies, and report the case herein together with literature review.

\section{CASE REPORT}

A 16-year-old boy visited an orthopedic hospital for pain in the right inguinal area that occurred after he fell down while standing on his hand during a physical exercise class, but as the simple X-ray did not show abnormal findings, the patient was observed with conservative management. Two days after the onset of symptoms, the patient had muscular weakness and hypesthesia on the right lower leg, so he underwent lumbar spine computed tomography (CT) at a general hospital, was hospitalized and treated conservatively under suspicion of spinal cord injury. Fourteen days after the onset of symptoms, since there was no improvement in his symptoms, the patient was transferred to the Department of Orthopedic Surgery in our hospital. He had no significant medical or trauma history before injury.

The simple X-ray of lumbar vertebrae and pelvic bone did not show abnormal findings. There was no abnormality on the thoracolumbar spine MRI except a mild herniated intervertebral disc between L5 and S1 and unilateral spondylolysis accompanied by spina bifida occulta.

Seventeen days after the onset of symptoms, the patient was referred to our Department of Rehabilitation Medicine for the assessment of muscular weakness in the right lower extremity. On physical examination, muscular atrophy was observed as the circumference of the right thigh measured at $10 \mathrm{~cm}$ proximal to the patel-
Table 1. Findings of Motor Nerve Conduction Study

\begin{tabular}{lccc}
\hline \multicolumn{1}{c}{ Nerve } & $\begin{array}{c}\text { Latency } \\
(\mathbf{m s})\end{array}$ & $\begin{array}{c}\text { Amplitude } \\
\text { (mV) }\end{array}$ & $\begin{array}{c}\text { Velocity } \\
\text { (m/s) }\end{array}$ \\
\hline SD \#17 & & & \\
Rt. common peroneal & 3.4 & 7.8 & 48 \\
Lt. common peroneal & 3.9 & 7.0 & 46 \\
Rt. tibial & 3.8 & 20.7 & 49.2 \\
Lt. tibial & 4.0 & 18.6 & 46 \\
Rt. femoral & 7.5 & 0.8 & \\
Lt. femoral & 6.7 & 12.0 & \\
SD \#82 & & & \\
Rt. femoral & 7.5 & 2.0 & \\
\hline
\end{tabular}

SD: Day after symptom developed

lar upper margin was $40 \mathrm{~cm}$ while that of the left thigh was $46 \mathrm{~cm}$ (Fig. 1-A), and severe tenderness along with ecchymosis was observed in the right inguinal area. According to the results of the manual muscle test (MRC, Medical Research Council), the left lower extremities were normal but the right lower extremities showed hip flexion $3 / 5$, knee extension $2 / 5$, ankle dorsiflexion $5 / 5$, great toe extension 5/5 and ankle plantar flexion 5/5. On sensory tests, hypesthesia and paresthesia were observed in the right anteromedial and anterolateral thigh, and the medial side of the lower leg (Fig. 1-B). The deep tendon reflex of the right knee also decreased.

On motor nerve conduction study, the amplitude of compound muscle action potential on the right femoral nerve decreased to $0.8 \mathrm{mV}$ compared to $12.0 \mathrm{mV}$ on the left side (Table 1). Sensory nerve conduction study for lateral femoral cutaneous nerve stimulated $1 \mathrm{~cm}$ inferior part of the anterior superior iliac spine (ASIS), and active electrodes attached on the $17 \mathrm{~cm}$ distal to the ASIS along the virtual line connecting between the ASIS and the patellar lateral margin. According to the results, the amplitude of sensory nerve action potential decreased to 
Table 2. Findings of Sensory Nerve Conduction Study

\begin{tabular}{lcc}
\hline \multicolumn{1}{c}{ Nerve } & $\begin{array}{c}\text { Latency } \\
(\mathbf{m s})\end{array}$ & $\begin{array}{c}\text { Amplitude } \\
(\mu \mathbf{V})\end{array}$ \\
\hline SD \#17 & & \\
Rt. superficial peroneal & 2.0 & 12 \\
Lt. superficial peroneal & 1.6 & 12 \\
Rt. sural & 1.8 & 16 \\
Lt. sural & 2.0 & 15 \\
Rt. lateral femoral cutaneous & 2.0 & 5 \\
\hline Lt. lateral femoral cutaneous & 1.7 & 17 \\
SD \#82 & & \\
Rt. lateral femoral cutaneous & 1.9 & 6 \\
\hline
\end{tabular}

SD: Day after symptom developed
$5 \mu \mathrm{V}$ on the right side compared to $17 \mu \mathrm{V}$ on the left side (Table 2). During somatosensory evoked potential study, we stimulated lateral femoral cutaneous nerves at $17 \mathrm{~cm}$ distal to the ASIS and used $1 \mathrm{~cm}$ long cutaneous needle electrodes, inserting active electrodes at $\mathrm{Cz}^{\prime}$ and reference electrodes at $\mathrm{Fz}$ in the international 10/20 system. While P37 latency was $30.5 \mathrm{msec}$ and N45 latency 38.1 msec on the left side, no response was observed on the right side. On needle electromyography (EMG), abnormal spontaneous activities were observed at rest in right iliopsoas, rectus femoris, vastus medialis and vastus lateralis muscles, and reduced recruitment of motor unit action potentials was observed during muscle contraction. Other lower limb and paravertebral muscles were normal

Table 3. Findings of Needle Electromyography

\begin{tabular}{|c|c|c|c|c|}
\hline Muscle & IA & ASA & MUAP morphology & Recruitment \\
\hline \multicolumn{5}{|l|}{ SD \#17 } \\
\hline Rt. Iliopsoas & Increased & ++ & Normal & Reduced \\
\hline Rt. Vastus lateralis & Increased & ++ & Normal & Reduced \\
\hline Rt. Vastus medialis & Increased & ++ & Normal & Reduced \\
\hline Rt. Rectus femoris & Increased & ++ & Normal & Reduced \\
\hline Rt. Adductor longus & Normal & 0 & Normal & Full \\
\hline Rt. Biceps femoris & Normal & 0 & Normal & Full \\
\hline Rt. Tensor fasciae latae & Normal & 0 & Normal & Full \\
\hline Rt. Gastrocnemius & Normal & 0 & Normal & Full \\
\hline Rt. Peroneus longus & Normal & 0 & Normal & Full \\
\hline Rt. Tibilais anterior & Normal & 0 & Normal & Full \\
\hline \multicolumn{5}{|l|}{ SD \#82 } \\
\hline Rt. Iliopsoas & Increased & ++ & Polyphasic & Reduced \\
\hline Rt. Vastus lateralis & Increased & ++ & Polyphasic & Reduced \\
\hline Rt. Vastus medialis & Increased & ++ & Polyphasic & Reduced \\
\hline Rt. Rectus femoris & Increased & ++ & Polyphasic & Reduced \\
\hline
\end{tabular}

IA: Insertional activity, ASA: Abnormal spontaneous activity, MUAP: Motor unit action potential

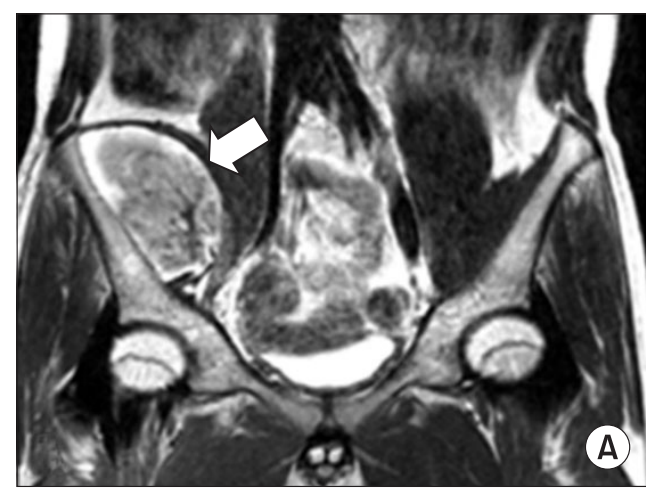

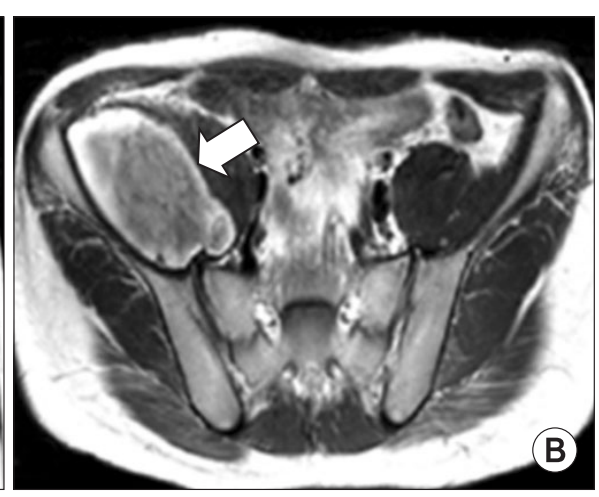

Fig. 2. Enhanced coronal (A) and transverse (B) T2-weighted pelvis MRI show an area of high signal intensity in the right iliacus muscle, consistent with hematoma (arrow). 
(Table 3).

Through electrodiagnostic study, we diagnosed the case as femoral neuropathy and accompanying meralgia paresthetica and performed pelvis MRI in order to identify the cause and locate the lesion. On pelvis MRI, a $9 \times 5 \times 4.5$ $\mathrm{cm}$ large hematoma was found in the right iliacus muscle and was removed through suction drainage on day 23 after the onset of symptoms (Fig. 2-A, B).

Eighty-two days after the onset of symptoms, the results of manual muscle testing (MRC, Medical Research Council) were right hip flexion $4 / 5$ and knee extension $3 / 5$, showing gradual recovery, but while sensory test showed recovery by around $20 \%$, paresthesia continued. On motor nerve conduction study, the amplitude of compound muscle action potentials for right femoral nerves increased to $2.0 \mathrm{mV}$, but on sensory nerve conduction study, the amplitude of sensory nerve action potentials for right lateral femoral cutaneous nerves did not noticeably change. On needle EMG, poly-phasic action potentials were observed in right iliopsoas, rectus femoris, vastus medialis and vastus lateralis muscles.

The results of manual muscle testing (MRC, Medical Research Council) performed 120 days after the onset of symptom at outpatient follow-up showed that muscle strength improved further to right hip flexion $4 / 5$ and knee extension $4 / 5$, and the results of sensory tests on that day were not different from those on day 82. Functionally, the patient was able to walk by himself both indoors and outdoors.

\section{DISCUSSION}

The femoral nerve arises from the posterior branch of the ventral rami of the L2-L4 roots, descends through the psoas muscle, passes between the psoas and iliacus groove and exits the pelvis below the inguinal ligament, in close proximity to the femoral artery and vein. The femoral nerve exits the pelvis under the inguinal ligament into the femoral triangle, where it produces sensory innervations to the anteromedial thigh and medial calf and sends off motor branches to the iliacus, sartorius, pectineus and quadriceps muscles.

Subsequently, femoral neuropathy is a disease clinically characterized by weakness of the iliopsoas muscle, numbness, loss of knee-jerk, and sensory loss of anteromedial thigh. ${ }^{1}$ As an uncommon peripheral neuropathy, it is usually caused by the compression of nerves around the inguinal area among several factors. ${ }^{2,6}$ Goodfellow et al. $^{7}$ performed an experiment injecting fluid into the iliacus using a cadaver, demonstrated a rigid fibrous arch in iliacus muscle which femoral nerves pass and found that the nerve can be compressed by the expansion of the iliopsoas muscle. Later Nobel et al. ${ }^{8}$ also demonstrated the same results by an experiment injecting colored latex into the iliacus muscle. Electrodiagnostic study and needle EMG for femoral nerves are useful in diagnosis. ${ }^{2}$

Lateral femoral cutaneous nerves mainly originate from the L2-L4 nerve roots and supply the anterolateral aspect of the thigh from the level of the inguinal ligament to the region of the knee. The nerve penetrates the psoas muscle, passes in front of the iliacus muscle, runs toward the ASIS and lateral attachments of the inguinal ligament and then pierces the fascia lata $10 \mathrm{~cm}$ below the inguinal ligament. When lateral femoral cutaneous nerves are injured, meralgia paresthetica occurs, inducing paresthesia, hypesthesia, numbness and pain in the anterolateral thigh without muscular weakness. ${ }^{3}$ Because of their long running distance and various atypical running routes, situations such as nerve entrapment may be induced in various sites but because of the anatomical characteristics, compression in the inguinal ligament occurs commonly. Nerve injury can be diagnosed through nerve conduction or somatosensory evoked potential studies. ${ }^{4}$

The accurate incidence of iliacus hematoma, which induced two types of neuropathy in our case, has not been reported, but can appear as a complication of anticoagulant, hemophilia, kidney transplantation or trauma. ${ }^{5-7}$ In general, it happens spontaneously in cases such as blood coagulation disorder, but is rarely induced by a minor injury in the pelvic muscle, and sometimes hip hyperextension or strong contraction becomes the cause of injury. ${ }^{6,9}$ In the case of our patient, the most likely cause of hematoma was the trauma that he had when falling down while standing on his hand. The patient's iliacus hematoma enlarged to $9 \times 5 \times 4.5 \mathrm{~cm}$, and it dislocated the psoas muscle to the inside. When hematoma increases pressure on the iliopsoas section, it compresses nerves running through the pelvis and may induce neuropathy in some cases. ${ }^{7,8}$ Neurological symptoms such as paresthesia and muscular weakness appear, and there are pain in the inguinal area as a primary symptom resulting from iliac muscle bleeding, consequent limited hip flexion and ex- 
tension, and compensatory bending deformation of the hip due to pressure on the iliac muscle and the expansion of fascia. ${ }^{1,9,10}$ When a patient complains of symptoms as above, definite diagnosis can be made by examining hematomas and structures around the pressed part through imaging techniques such as ultrasound, CT and MRI, but ultrasonic diagnosis is sometimes difficult because muscle is situated deeply and covered by the intestinal tract, and MRI is the best choice in order to measure the accurate size of the hematoma or to identify pressed nerves and muscles., 9

The treatment of the disease may be different according to the severity of symptoms and underlying diseases and is largely divided into conservative treatment and surgery. As it is not clear which is the best, the disease is approached according to procedure for other types of pressure neuropathy. If the hematoma is small in size or there is a evidence for clinical improvement, conservative treatments such as bed rest, the correction of blood coagulation disorder and transfusion are preferred. ${ }^{5,7}$ However, if there is no improvement despite such conservative treatments, if neurological symptoms progress or if MRI clearly shows nerve compression by hematoma, surgical treatments such as hematoma evacuation and percutaneous drainage need to be considered. ${ }^{4,5}$ Prognosis depends on the intensity and period of nerve compression, but recovery takes several days to several months and most cases are reported to be recovered within 6 months. ${ }^{5}$

In conclusion, our case is a rare example showing that iliacus hematoma can occur by direct trauma and induce associated pressure neuropathy, and is characteristic in two aspects. First, it has occasionally been reported that iliacus hematoma occurs spontaneously due to the use of anticoagulants or in hemophilia, ${ }^{5-7}$ but has very rarely been reported that it occurs by a trauma, namely, a direct external impact. ${ }^{6}$ Second, the hematoma occurring in the patient's right iliac muscle pressed both femoral and lateral femoral cutaneous nerve on the same side and induced two types of neuropathy, femoral neuropathy and meralgia paresthetica, simultaneously, which is also a very rare phenomenon. Therefore, we report such an uncommon case together with the findings of related neurologic examinations, MRI and electrodiagnostic studies.

\section{REFERENCES}

1. Jenkins DB. Hollinshead's functional anatomy of limbs and back, 9th ed, Philadelphia: WB Saunders, 2009, 255-278

2. Kim MO, Kwon HK. Femoral mononeuropathy induced by malignant fibrous histiocytoma in retroperitoneal cavity. J Korean Acad Rehab Med 1994; 18: 437 442

3. Aszmann OC, Dellon ES, Dellom AL. Anatomical course of the lateral femoral cutaneous nerve and its susceptibility to compression and injury. Plast Reconstr Surg 1997; 100: 600-604

4. Nahabedian MY, Dellon AL. Meralgia paresthetica: etiology, diagnosis, and outcome of surgical decompression. Ann Plast Surg 1995; 35: 590-594

5. Rochman AS, Vitarbo E, Levi AD. Femoral nerve palsy secondary to traumatic pseudoaneurysm and iliacus hematoma. J Neurosurg 2005; 102: 382-385

6. Lee SH, Kim KH, Jo GY. Bilateral femoral neuropathy secondary to unilateral retroperitoneal hematoma. J Korean Acad Rehab Med 2001; 25: 168-172

7. Goodfellow J, Fearn CB, Matthews JM. Iliacus haematoma. A common complication of haemophilia. J Bone Joint Surg Br 1967; 49: 748-756

8. Nobel W, Marks SC Jr, Kubik S. The anatomical basis for femoral nerve palsy following iliacus hematoma. J Neurosurg 1980; 52: 533-540

9. Kang BS. Acute femoral neuropathy secondary to iliacus hematoma. J Korean Soc Emerg Med 2006; 17: 361-365

10. Seijo-Martineza M, Castro del Rio M, Fontoirab E, Fontoirac M. Acute femoral neuropathy secondary to an iliacus muscle hematoma. J Neurol Sci 2003; 209: 119-122 